research is to: a) Identify approaches to supporting evidence informed decision-making in health systems serving Syrian refugees, b) understand some of the barriers and facilitators to using these approaches in health systems serving the Syrian refugee crisis. The first study of its type done as an integrated KT, where it was completed in close partnership with the ultimate users Médecins Sans Frontières, also known as Doctors Without Borders (MSF).

Background: Providing essential healthcare is becoming a huge undertaking requiring a multifaceted approach for over four million Syrian refugees. The scarcity of available resources makes it imperative that resources are used based on research evidence, to maximize the health outcomes among vulnerable populations. The challenge is that there is still a gap on how to best utilize research evidence to inform decision making in the field.

Methods: Document analysis and key informant interviews utilizing semi-structured questions at Médecins Sans Frontières, to identify some of the barriers and facilitators by using Knowledge Translation (KT) approaches in health systems serving the Syrian refugee crisis.

Results: Facilitators to MSF's use of research evidence in decision-making include MSF uses surveys to assess and identify research gaps in the field. Barriers to MSF's use of research evidence in decision-making include lack of a receptive climate for research remains a barrier to the utilization of research knowledge in decision-making and lack of a formalized process for field staff to acquire research evidence.

Conclusion: Understanding the findings of the above research questions would enhance the quality, effectiveness and coverage of healthcare programme delivery for Syrian refugees and enable the health system to be more responsive to the healthcare needs of the Syrian refugees.

Prehosp Disaster Med 2017;32(Suppl. 1):s53-s54

doi:10.1017/S1049023X17001492

\section{Verification of an Area Disaster Resilience Management System Model for Healthcare During the 2016 Kumamoto Earthquake Masabiko Munechika ${ }^{1}$, Chisato Kajibara ${ }^{1}$, Masataka Sano ${ }^{2}$, Masaaki Kaneko ${ }^{3}$, Haizhe Jin ${ }^{4}$ \\ 1. Waseda University, Tokyo/Japan \\ 2. Chiba Institute of Technology, Chiba/Japan \\ 3. Tokai University, Tokyo/Japan \\ 4. Northeastern University, Liaoning/China}

Study/Objective: The research group is developing an Area Disaster Resilience Management System Model for Healthcare (ADRMS-H), composed of municipal and health care organizations, to enhance the health care resilience of a community (Figure 1). This model is an extended form of a Business Continuity Management System for a single organization. We are introducing the model to Kawaguchi City in Saitama Prefecture. In this study, we investigate successes and failures of disaster medicine during the Kumamoto Earthquake in April 2016, with the intent to verify the ADRMS-H model.
Background: Japan faces a high risk of natural disasters such as earthquakes, during which it is urgent that countermeasures are taken to secure business continuity. To enhance the health care resilience of a community, ADRMS-H must be established.

Methods: We interviewed the medical staff of the Japanese Red Cross Kumamoto Hospital, and other disaster-based hospitals, to investigate successes and failures of disaster medicine performed during the Kumamoto Earthquake. We also interviewed medical assistance teams, such as the Disaster Medical Assistance Team (DMAT), the Disaster Psychiatric Assistance Team (DPAT), and so on. We investigated if a function to achieve a positive result or a function to overcome negative results has been incorporated in the ADRMS-H.

Results: In disaster medicine during the Kumamoto Earthquake, "information collection," "chain of command," and "provision of relief supplies" were the main failures. The functions to overcome these issues have already been incorporated in the ADRMS-H model. On the other hand, the successes of assistance by the DMAT command headquarters outside of the disaster area (Tokyo and Osaka in this case) were effective. The organizational plan and function to achieve similar success in the future have not been incorporated.

Conclusion: We confirmed that the ADRMS-H model is valid for disaster management, and, to improve it, we must add a medical assistance team headquarters.

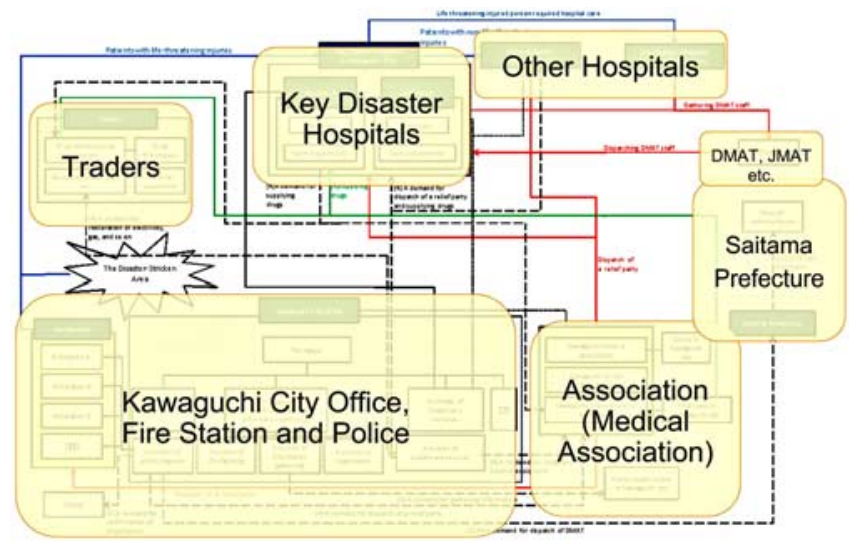

Prehosp Disaster Med 2017;32(Suppl. 1):s54 doi:10.1017/S1049023X17001509

Changes in the Functions for Continued Healthcare Services during a Disaster Chisato Kajihara ${ }^{1}$, Masabiko Munechika ${ }^{1}$, Masataka Sano ${ }^{2}$, Masaaki Kaneko ${ }^{3}$, Haizhe Jin ${ }^{4}$

1. Waseda University, Tokyo/Japan

2. Chiba Institute of Technology, Chiba/Japan

3. Tokai University, Tokyo/Japan

4. Northeastern University, Liaoning/China

Study/Objective: This study aims to identify the organizational functions that are needed to ensure continued health care services during a disaster. Moreover, this paper reveals the roles 